\title{
SONDA DE TDR PARA A ESTIMATIVA DE UMIDADE EM BAGAÇO DE
} CANA-DE-AÇÚCAR

Doi:http://dx.doi.org/10.1590/1809-4430-Eng.Agric.v36n 1p24-35/2016

\section{CLAUDINEI F. SOUZA ${ }^{1}$, LUCAS DA S. CIRILO ${ }^{2}$, REINALDO G. BASTOS ${ }^{3}$, JOSÉ G. PERES ${ }^{4}$, ALINE F. OLIVEIRA ${ }^{5}$}

\begin{abstract}
RESUMO: O bagaço de cana-de-açúcar pode ser utilizado como suporte sólido para cultivo de microrganismos, e apesar de fundamental, o monitoramento da umidade em tempo real nesses processos ainda é pouco pesquisado. Sendo assim, o presente trabalho teve como objetivo propor e validar a TDR para estimar a umidade em bagaço de cana-de-açúcar, visando a seu uso como suporte para cultivo em estado sólido, considerando a produção de ácido cítrico. A sonda de TDR proposta foi analisada fisicamente, e, posteriormente, para a validação dos resultados, realizou-se uma calibração com leituras da constante dielétrica do bagaço de cana-de-açúcar até à saturação do material, os quais foram comparados com o método-padrão e equação universal de Topp. Também, foram conduzidas em colunas de leito fixo preenchidas com bagaço a $80 \%$ de umidade, com monitoramento da umidade através de sonda de TDR para validar a técnica. Pôde-se verificar que existe viabilidade no uso da técnica da TDR para estimar a umidade volumétrica, e com o auxílio de ferramentas gráficas e regressão linear, foi proposta a equação de calibração: $(\theta=0,0253 * \mathrm{Ka}$ 0,0025). Com isso, pode-se controlar e monitorar in situ a umidade do bagaço de cana-de-açúcar, através da técnica da TDR.
\end{abstract}

PALAVRAS-CHAVE: TDR, monitoramento de umidade, cultivo em estado sólido, Aspergillus Níger, constante dielétrica.

\section{TDR PROBE FOR BAGASSE MOISTURE MEASUREMENT}

\begin{abstract}
Bagasse sugarcane can be used as a solid substrate for microorganism cultivation. Despite fundamental, moisture real-time monitoring of these processes is still poorly investigated. Thus, this study aimed at developing and validating a TDR probe to estimate moisture in sugarcane bagasse, which is used as cultivation mean of microorganisms considering citric acid production. The proposed probe was physically evaluated and then used to validate the results. Calibration readings of the bagasse dielectric constant were taken up to its saturation, and subsequently compared to standard method and Topp's universal equation. In addition, we carried out studies in fixed-bed columns filled with bagasse at $80 \%$ moisture, monitoring its moisture through TDR probe, so we could validate the technique. We concluded such technique feasibility to estimate water content. Furthermore, we proposed a calibration equation using graphical tools and linear regression $(\theta=0.0253 * \mathrm{Ka}-0.0025)$. As a result, we could then control and monitor bagasse sugarcane moisture in situ by the TDR technique.
\end{abstract}

KEYWORDS: TDR, moisture monitoring, solid-state cultivation, Aspergillus niger, dielectric constant.

\footnotetext{
${ }^{1}$ Eng $^{\circ}$ A grônomo, Prof. Doutor, Departamento de Recursos Naturais e Proteção Ambiental, UFSCar/Araras - SP, Fone: (19) 3543-2616,cfsouza@cca.ufscar.br

${ }^{2}$ Eng $^{\circ}$ A grônomo, Departamento de Recursos Naturais e Proteção Ambiental, UFSCar/Araras - SP, Fone: (19) 3543-2616, lucas.cirilo@cacaushow.com.br

${ }^{3}$ Eng $^{\circ}$ de Alimentos, Prof. Doutor, Departamento de Tecnologia A groindustrial e Sócio economia Rural, UFSCar/Araras - SP, Fone: (19) 3543-2614, reinaldo@cca.ufscar.br

${ }^{4}$ Eng $^{\circ}$ A grônomo, Prof. Doutor, Departamento de Recursos Naturais e Proteção Ambiental, UFSCar/Araras - SP, Fone: (19) 3543-2616,jogepe@cca.ufscar.br

${ }^{5}$ Biotecnologista, Departamento de Tecnologia Agroindustrial e Sócio economia Rural, UFSCar/Araras - SP, Fone: (19) 3543-2614. 


\section{INTRODUÇÃO}

A cultura da cana-de-açúcar, quando utilizada para a produção de açúcar e álcool, gera subprodutos ou resíduos como o bagaço, palha, melaço, torta de filtro e vinhaça, os quais hoje têm grande importância econômica. O bagaço, por exemplo, vem sendo utilizado como combustíveis, biomassa, veículo para ração animal, substrato para crescimento microbiano, entre outros (FASANELLA, 2008).

O cultivo de microrganismos em estado sólido tem apresentado grande potencial tecnológico para produção de alimentos, produtos químicos e farmacêuticos. O processo caracteriza-se pelo crescimento de microrganismos numa matriz sólida, ou seja, a partir de um substrato insolúvel, na ausência ou perto da ausência de água livre (SINGHANIA et al., 2009). Comparado com os cultivos microbianos submersos, o meio sólido apresenta uma fase gasosa e baixa quantidade de água entre as partículas, sendo utilizados biorreatores agitados ou estáticos, como colunas de leito fixo (BREUKELEN et al., 2011).

A matriz sólida pode ser um substrato natural contendo fonte de carbono, ou um suporte inerte impregnado de solução nutriente. Os suportes utilizados nos processos em estado sólido podem variar em sua composição, tamanho, resistência mecânica, porosidade e capacidade de retenção de água (SINGHANIA et al., 2009). Nesse sentido, resíduos agroindustriais, como farelo de trigo, de milho e bagaço de cana-de-açúcar, servem como fonte de nutrientes e suporte para esses processos na medida em que pos suem características poliméricas e são insolúveis em água.

O bagaço de cana-de-açúcar vem sendo proposto como suporte sólido para obtenção de ácido cítrico a partir de Aspergillus niger. Nesse sentido, um dos parâmetros mais importantes de controle e de monitoramento nos cultivos em estado sólido é a umidade do meio, medida que normalmente não é acompanhada continuamente durante o processo. Especificamente em cultivos com Aspergillus niger, a umidade deve estar em torno de 65 a $75 \%$ para garantir um desenvolvimento adequado, produtividade e rendimentos competitivos com os processos microbianos submersos (KUMAR \& JAIN, 2008).

A medida da umidade de materiais biológicos sólidos, convencionalmente, implica a modificação das características físicas do material. Além disso, como os substratos sólidos utilizados podem sofrer degradação, essa medida deve ser obtida o mais breve possível (VALERO et al., 2009).

A metodologia-padrão é obtida dividindo-se a massa de água contida na amostra pela massa de suas partículas sólidas, ou seja, da sua massa seca. A massa seca é obtida secando-se a amostra em estufa até massa constante (HORWITZ \& LATIMER, 2011). Entretanto, esse monitoramento no caso dos processos microbianos em estado sólido envolve a retirada de amostra e a interferência direta nos biorreatores, além de modificação do volume reacional e da geometria do leito de partículas.

Dessa forma, uma medida de umidade do meio sólido durante o crescimento microbiano minimizaria uma das maiores dificuldades encontradas nos cultivos em estado sólido, ou seja, o monitoramento e o controle de parâmetros de processo. Esta inovação tecnológica pode representar o desenvolvimento de novas configurações de biorreatores que permitam a manutenção da umidade em níveis ótimos para os microrganismos, levando à obtenção de altos rendimentos e de produtividades. Além disso, o monitoramento em linha da umidade pode ser correlacionado a outros parâmetros, servindo ainda como estimativa do crescimento celular, outro ponto crítico nestes processos (BASTOS, 2006).

Dos vários métodos existentes, a técnica da TDR - "Time Domain Reflectometry" - vem despertando muito interesse devido principalmente à possibilidade de leituras em tempo real de forma automatizada (SANTORO et al., 2008).

A utilização dessa técnica tem-se expandido e proporcionado uma contribuição relevante para os estudos da relação solo-água-planta-atmosfera, possibilitando, dentre outros, avaliar a 
condutividade elétrica do solo (SONCELA et al., 2011), identificar o perfil de extração de água do solo pelo sistema radicular das culturas (BRUNHARA et al., 2011), estimar a evapotranspiração das culturas (OLIVEIRA NETO et al., 2011) e analisar o movimento de água no solo (SOUZA \& FOLEGATTI, 2010; LOPES et al., 2010; BARROS et al., 2010).

Para estimativas precisas de umidade utilizando o TDR, recomenda-se o ajuste de uma curva de calibração para cada tipo de material analisado, um inconveniente que normalmente é aceitável devido às vantagens que apresenta em relação a outras técnicas (SOUZA et al., 2009). Outra desvantagem da utilização desta técnica tem sido o custo ainda alto para os padrões nacionais (ELAIUY et al., 2009).

A TDR baseia-se no efeito da umidade do solo sobre a velocidade de propagação de pulsos de micro-ondas em cabos condutores envoltos por solo. Este fenômeno deve-se à diferença entre as constantes dielétricas (K [adimensional]) da água, do ar e do material sólido do solo. Na matriz do solo, as constantes dielétricas variam entre 1 e 81 . O ar possui o valor mínimo igual a 1 , as partículas sólidas variam entre 3 e 5, e a água possui o valor máximo: 81 (SANTORO et al., 2008; SILVA et al., 2012). A constante K, medida no sistema solo-água-atmosfera, é denominada de constante dielétrica aparente $(\mathrm{Ka})$; assim, conhecendo-se essa variável, pode-se determinar a umidade volumétrica do solo por meio de curvas de calibração obtidas a partir de dados de campo ou de laboratório.

Desta forma, o objetivo deste trabalho foi propor e validar a técnica da TDR para estimar a umidade em bagaço de cana-de-açúcar, visando a seu uso como suporte sólido para obtenção de ácido cítrico.

\section{MATERIAL E MÉTODOS}

Este experimento foi realizado nas dependências do Laboratório de Hidráulica Agrícola do Centro de Ciências Agrárias da Universidade Federal de São Carlos (CCA/UFSCar), localizado na cidade de Araras - SP. Na calibração, foi utilizada uma sonda de TDR do tipo coaxial projetada e idealizada para a estimativa da umidade em substratos (Plantmax ${ }^{\circledR}$ e Fibra de coco) de mudas florestais (ELAIUY et al., 2009). Partindo do pressuposto de que as características físicas dos substratos e do bagaço são parecidas, foi adotada a utilização do mesmo modelo de sonda. Suas dimensões são baseadas em uma célula de transmis são coaxial.

Segundo ELAIUY et al. (2009), o desenvolvimento das sondas levou em conta os critérios observados na literatura: 1) Um mínimo de sinal eletromagnético captado; 2) Impedância característica inferior a $200 \Omega$; 3) Uniformidade na distribuição de energia conduzida pela sonda no meio amostrado; 4) Mínimo custo.

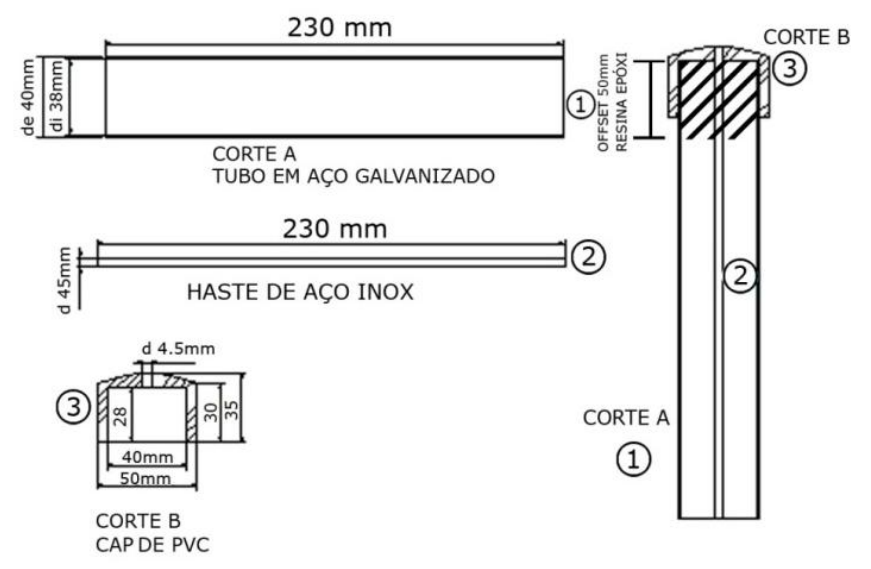

FIGURA 1. Detalhamento de construção da sonda (ELAIUY et al., 2009). Sche matic diagram of probe construction (ELAIUY et al., 2009). 
Para a construção da sonda coaxial, ELAIUY et al. (2009) descrevem que, primeiramente, foi torneada uma rosca na base de um cilindro de ferro galvanizado com 0,23 m de extensão e $0,04 \mathrm{~m}$ de diâmetro para que fosse rosqueado nesta extremidade um "cap" de PVC com 1 1 1/4" de diâ metro.

No centro desse "cap", foi feito um orifício para permitir a passagem de um cabo coaxial RG-58 de $50 \Omega$, o qual teve seu condutor interno soldado na extremidade inferior de uma haste em aço inox de 0,23 m de extensão e 0,0045 m de diâmetro, e sua malha externa foi seccionada em duas partes e soldadas em dois pontos na parede interna do cilindro. Na outra extremidade do cabo, foi colocado um conector BNC, a fim de conectar a sonda junto ao reflectômetro. Com o "cap" devidamente rosqueado e a haste já centrada no eixo axial do cilindro, a sonda foi presa num suporte vertical, e sua extremidade inferior foi preenchida com resina epóxi até que se atingisse uma altura de 0,05 $\mathrm{m}$ (Offset), a fim de fixar a haste.

O detalhamento da construção da sonda e suas dimensões são apresentados na Figura 1.

A sonda proposta, conforme a Figura 2, foi avaliada fisicamente através da impedância característica e da sensibilidade espacial, conforme descrito por SANTORO et al. (2008), sendo o resultado comparado com outros modelos de sondas de TDR, Sonda contínua e Sonda muti-haste.

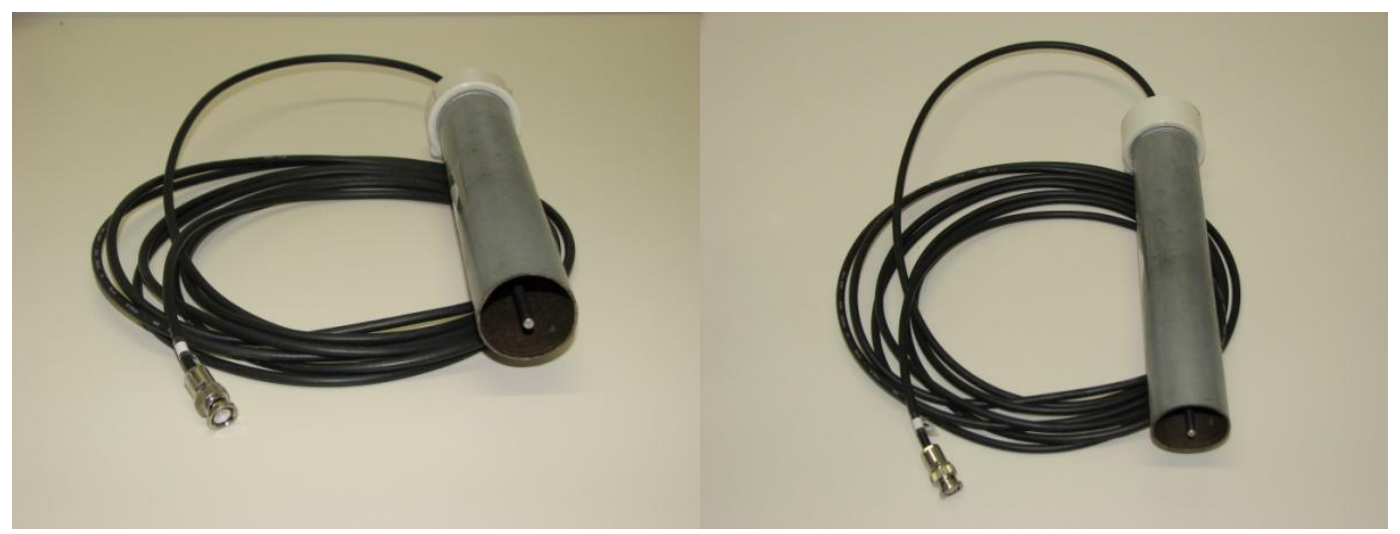

FIGURA 2. Sonda coaxial. Coaxial probe.

Para a realização das avaliações mencionadas, utilizou-se de um Reflectômetro TDR 100 - Campbell Scientific, equipado com uma interface RS 232, onde foi analisado o sinal eletromagnético, automaticamente, pelo programa WinTDR 6.0 (Utah State University, LoganUtah).

O bagaço utilizado foi coletado em usinas de açúcar e de álcool da região da cidade de Araras-SP, localizada a $170 \mathrm{~km}$ da capital do Estado de São Paulo.

Primeiramente, o bagaço de cana-de-açúcar a ser utilizado foi classificado em agitador mecânico com um conjunto de peneiras, sendo utilizadas as partículas retidas entre as peneiras TYLER 14 e 28, ou seja, diâmetros entre 0,59 e 1,17 mm.

Para a determinação da densidade úmida do bagaço de cana-de-açúcar, foi empregado o método descrito por HOFFMAN (1970), utilizado pela Federação dos Institutos para a Pesquisa e Análises Agrícolas da Alemanha (VDLUFA), para análise de substratos hortícolas (RÖBER \& SCHALLER, 1985).

Nessa metodologia, seis amostras do bagaço no estado fresco e já peneirado são colocadas em provetas de plástico transparente e graduadas, de $500 \mathrm{~mL}$ de capacidade (Figura 3), a qual se deixa cair por 10 vezes consecutivas sob ação de seu próprio peso, de uma altura de $10 \mathrm{~cm}$. Com ajuda de uma espátula, nivela-se o volume obtido $(\mathrm{mL})$. Em seguida, pesa-se o material fresco $(\mathrm{g})$ e colocase em estufa a $65^{\circ} \mathrm{C}$, por 48 horas ou até peso constante. Os valores das densidades de volume (média de três amostras) são obtidos aplicando-se a seguinte fórmula:

$$
\text { Densidade Úmida }\left(\mathrm{kg} \mathrm{m}^{-3}\right)=\frac{\operatorname{Massaúmida}(\mathrm{g})}{\operatorname{Volume}\left(\mathrm{cm}^{3}\right)} \text { x1000 }
$$




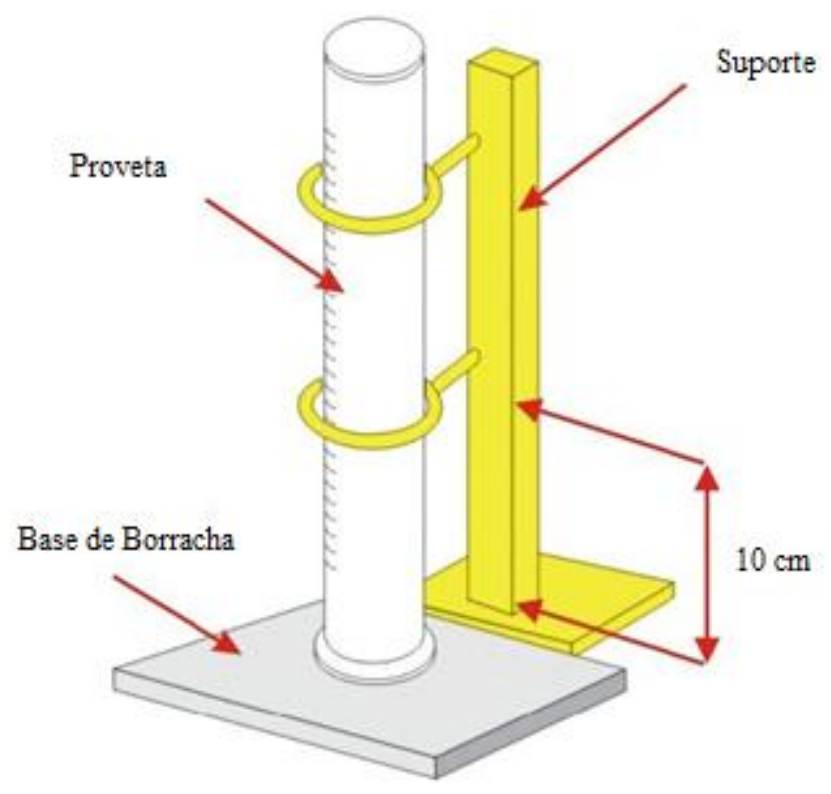

FIGURA 3. Arranjo utilizado para a determinação da densidade da amostra de bagaço de cana-deaçúcar. System used for sample density measurement.

Para efetuar as determinações da constante dielétrica aparente $(\mathrm{Ka})$ e da umidade volumétrica ( $\theta$ ) com a sonda coaxial, seguiu-se o procedimento descrito por ELAIUY et al. (2009). Nessa metodologia, as etapas de umedecimento, com adição de $50 \mathrm{~mL}$ de água por etapa, foram continuamente repetidas, até atingir umidades próximas à da saturação, conforme descrição: metade da amostra de bagaço coletada e peneirada, cerca de $2 \mathrm{~L}$, foi esparramada em uma mesa, formando uma fina camada.

Utilizando um pulverizador, adiciona-se cerca de metade da água desejada $(25 \mathrm{~mL})$; a seguir, a outra metade da amostra foi esparramada sobre a metade anterior, pulverizando-se com água até completar os $50 \mathrm{~mL}$. Após o umedecimento, a amostra deve ser bem homogeneizada, e, na sequência, o bagaço de cana-de-açúcar foi colocado em uma sonda coaxial, aproximadamente 0,22 L de volume, compactado de modo a manter sua densidade o mais uniforme possível, aproximadamente $150 \mathrm{~kg} \mathrm{~m}^{-3}$ (Figura 4).

A sonda foi conectada ao reflectómetro, e foram lidos os valores de umidade volumétrica ( $\theta$ Topp) e de constante dielétrica aparente $(\mathrm{Ka})$ para cada umedecimento. Em seguida, uma amostra de bagaço foi colocada em uma cápsula e pesada, sendo seca em estufa a $105^{\circ} \mathrm{C}$, por 24 horas, para a obtenção da umidade gravimétrica (método-padrão), AOAC (2005). As estimativas obtidas foram comparadas com o método-padrão (gravimétrico), utilizando um diagrama de dispersão e coeficiente de correlação.

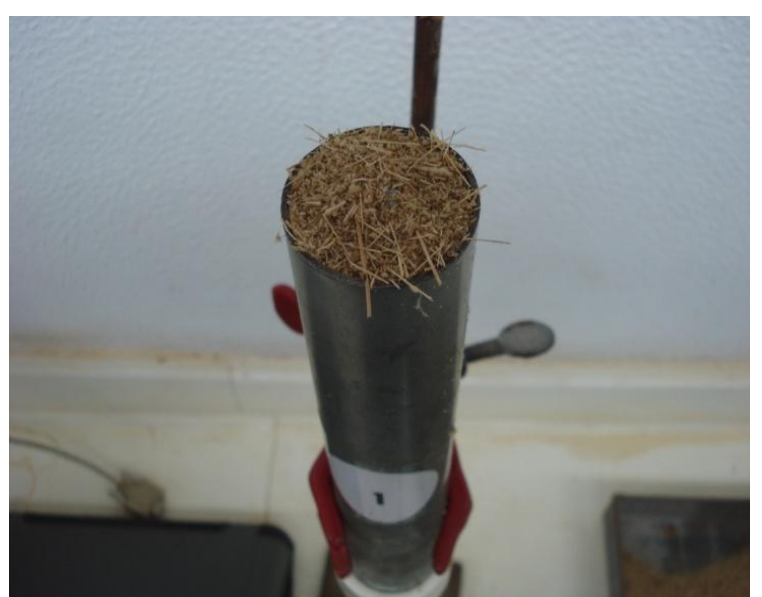

FIGURA 4. Sonda coaxial com o bagaço de cana-de-açúcar. Coaxial probe filled with sugarcane bagasse. 
Para a validação da calibração da sonda de TDR realizada anteriormente, o bagaço de cana-de-açúcar teve seu teor de água monitorado durante o cultivo em estado sólido de Aspergillus niger. Os experimentos foram conduzidos em coluna de leito fixo, no Laboratório de Microbiologia Aplicada (LABMAC/CCA/UFSCar), sendo preenchidos $6 \mathrm{~cm}$ da coluna com o suporte sólido, a uma temperatura de $25^{\circ} \mathrm{C}, 0,4 \mathrm{~L} \mathrm{~min}^{-1}$ de vazão de ar saturado de água por umidificador, como representa a Figura 5, com monitoramento por seis dias.

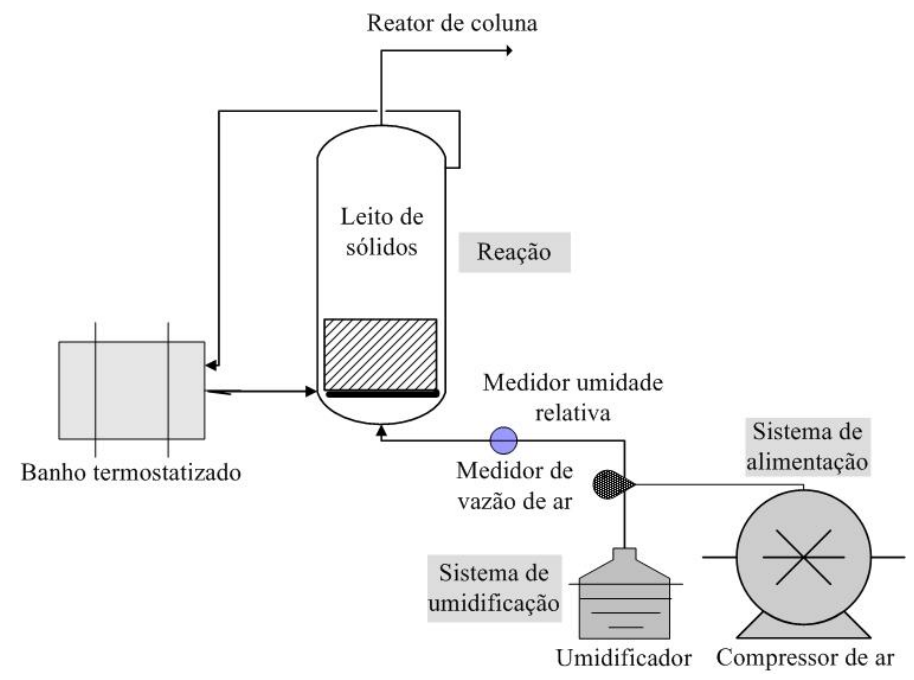

FIGURA 5. Esquema dos cultivos em estado sólido de Aspergillus niger em bagaço de cana-deaçúcar impregnado com vinhaça (adaptado de BASTOS, 2006). Diagram of solidstate medium for Aspergillus niger cultivation using sugarcane bagasse with vinasse (adapted from BASTOS, 2006).

A umidade do bagaço de cana-de-açúcar foi monitorada em linha durante os cultivos, diretamente nas colunas de leito fixo, pela técnica de Reflectometria no Domínio de Tempo (TDR), como mostra a Figura 6. Os biorreatores apresentavam uma sonda TDR do tipo contínua, de três hastes, para a medida da constante dielétrica dos sólidos, com estimativa da umidade através de equação linear de calibração.
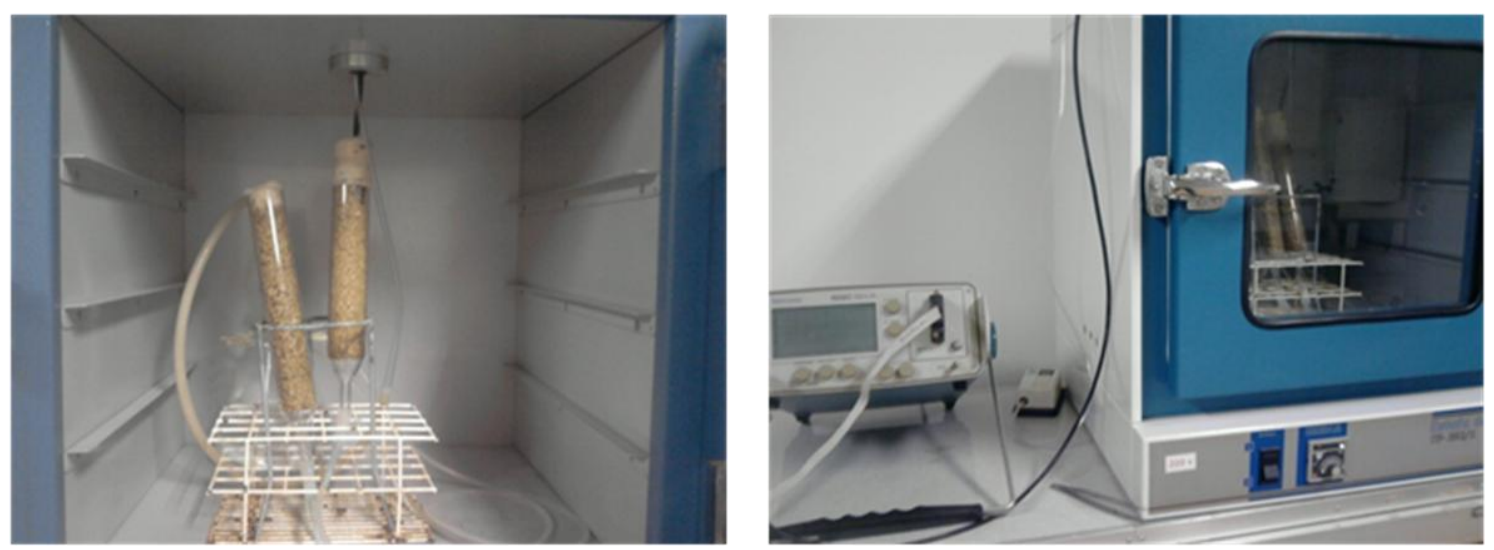

FIGURA 6. Processo de cultivo em estado sólido, utilizando sonda de TDR, para monitorar a umidade. Solid-state growth process using TDR probe as moisture monitor.

\section{RESULTADOS E DISCUSSÃO}

A Tabela 1 apresenta as dimensões utilizadas da sonda e os valores de impedância, tanto medida como calculada, inferior ao recomendado (200 $\Omega$ ) e com sensibilidade espacial $\geq 0,1$ (SANTORO et al., 2008). Os resultados observados demonstram que a sonda proposta possui características dimensionais apropriadas para atender às atribuições físicas desejadas em uma sonda de TDR, quando comparada com outros modelos de sondas. 
TABELA 1. Dimensões, impedância medida e calculada para diferentes configurações de sondas de TDR. Dimensions, measured and calculated impedance for different TDR-probe configurations.

\begin{tabular}{|c|c|c|c|c|c|c|}
\hline & \multicolumn{2}{|c|}{ Diâmetro $(\mathrm{m})$} & \multirow{2}{*}{$\frac{\text { Sensibilidade espacial }}{\mathrm{a} / \mathrm{b}}$} & \multirow{2}{*}{$\begin{array}{l}\text { Volume } \\
\qquad\left(\mathrm{m}^{3}\right)\end{array}$} & \multicolumn{2}{|c|}{ Impedância $(\Omega)$} \\
\hline & Interno a & $\begin{array}{c}\text { Externo } \\
\text { b }\end{array}$ & & & Calculada & Medida \\
\hline Sonda coaxial & 0,0045 & 0,040 & 0,11 & 0,0002 & 131,08 & 172,67 \\
\hline Sonda contínua & 0,0030 & 0,027 & 0,11 & 0,0001 & 131,83 & 197,00 \\
\hline Sonda muti-haste & 0,0060 & 0,058 & 0,11 & 0,0005 & 132,43 & 168,00 \\
\hline
\end{tabular}

A massa do bagaço de cana-de-açúcar seco em estufa, que completou o volume da sonda coaxial, foi de 33,92 g. Sabendo desse dado, pode-se aplicar a Eq. 1. Na Tabela 2, podem ser observados os valores da densidade do bagaço da cana-de-açúcar e também da densidade quando relacionada com a da água. Estes valores foram utilizados na determinação da umidade volumétrica pelo método-padrão do bagaço da cana-de-açúcar.

TABELA 2. Valores da densidade do material e da densidade relativa. Sugarcane bagasse density and relative density values.

\begin{tabular}{lcccc}
\hline Material & Massa $(\mathrm{g})$ & Volume $\left(\mathrm{cm}^{3}\right)$ & Densidade $\left(\mathrm{kg} \mathrm{m}^{-3}\right)$ & Densidade relativa (adimensional) \\
\hline Bagaço & 33,92 & 225 & 150,76 & 0,151 \\
\hline
\end{tabular}

Com os resultados numéricos das umidades apresentados na Tabela 3, foi feita a correlação entre os valores obtidos pelas duas técnicas, a fim de comparar o método-padrão com a técnica da TDR que utiliza a equação de Topp (S ANTOS et al., 2010). Para cada leitura foram realizadas 3 repetições, sendo o valor utilizado a média entre as repetições. A partir dos resultados de Ka e umidade volumétrica obtida por gravimetria, puderam determinar-se as equações representativas para as condições em estudo.

TABELA 3. Resultados da umidade volumétrica nos dois métodos e da constante dielétrica (Ka) do bagaço de cana-de-açúcar. Water content measured by both methods and dielectric constant ( $\mathrm{Ka}$ ) of suga rcane bagasse.

\begin{tabular}{cccc}
\hline \multirow{2}{*}{ Leituras } & Constante dielétrica $(\mathrm{Ka})$ & \multicolumn{2}{c}{ Umidade $\left(\mathrm{m}^{3} \mathrm{~m}^{-3}\right)$} \\
\cline { 3 - 4 } & 1,91 & 0,01 & $\theta$ (Topp) \\
\hline 1 & 3,54 & 0,05 & 0,001 \\
2 & 4,32 & 0,09 & 0,044 \\
3 & 5,54 & 0,12 & 0,063 \\
4 & 6,53 & 0,16 & 0,093 \\
5 & 7,92 & 0,20 & 0,115 \\
6 & 9,29 & 0,24 & 0,146 \\
7 & 11,39 & 0,30 & 0,174 \\
8 & 12,14 & 0,34 & 0,212 \\
9 & 14,01 & 0,39 & 0,228 \\
10 & 16,19 & 0,44 & 0,260 \\
11 & 19,63 & 0,51 & 0,294 \\
12 & 21,07 & 0,56 & 0,341 \\
13 & 25,71 & 0,63 & 0,358 \\
14 & 27,71 & 0,68 & 0,407 \\
15 & 28,85 & 0,75 & 0,425 \\
16 & 35,33 & 0,84 & 0,435 \\
17 & & & 0,482 \\
\hline
\end{tabular}


Como pôde ser observado, o bagaço de cana-de-açúcar apresentou ponto de saturação na $17^{\text {a }}$ leitura, ou seja, as leituras seguintes (18 e 19ª $)$ não alteraram o resultado da Ka por apresentar formação de lâmina água na amostra.

A Figura 8 apresenta relação entre os valores da umidade volumétrica ( $\theta$ Topp) e os da umidade volumétrica pelo método-padrão, a qual apresenta que a equação de Topp subestima a umidade do bagaço da cana-de-açúcar em aproximad amente $59 \%$.

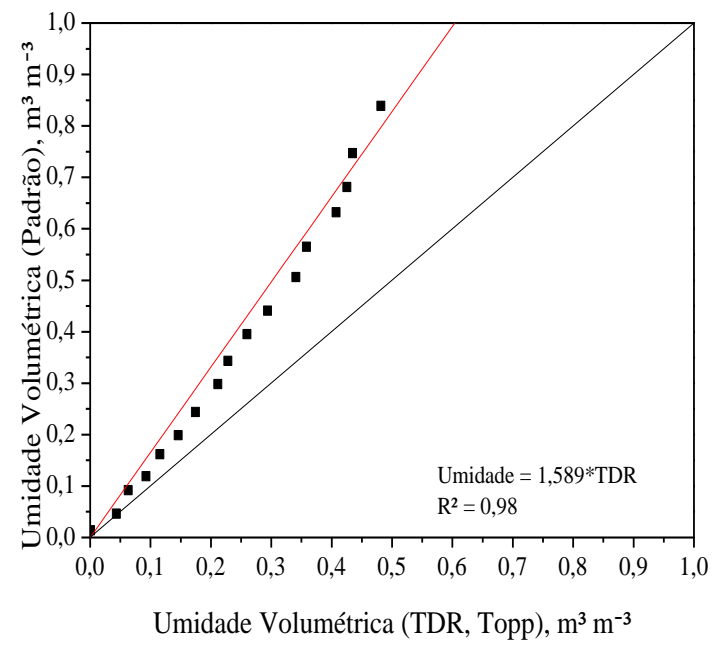

FIGURA 8. Relação entre o método-padrão e a equação de Topp. Comparison between standard method and Topp's equation.

O diagrama de dispersão comparando as duas técnicas demonstra que a água foi retida pelas partículas do bagaço de cana-de-açúcar e não permanece ou não se encontra mais livre neste meio. Isto implica uma discrepância entre os valores da umidade obtidos pela técnica da TDR e os valores obtidos pelo método-padrão. De acordo com VALERO et al. (2009), a afirmativa encontrada na literatura especializada, de que a constante dielétrica aparente dos meios porosos depende apenas de seu conteúdo de água, pode estar equivocada.

Em estudos realizados para a avaliação da umidade em diferentes substratos, ELAIUY et al. (2009) comprovam que os materiais avaliados apresentaram distintas umidades volumétricas quando submetidos à avaliação por este modelo, sendo de se esperar que tivesse o mesmo tipo de comportamento para todos os substratos, demonstrando que a constante dielétrica aparente (Ka) depende das propriedades intrínsecas de cada material.

Os resultados deste trabalho, juntamente com os estudos realizados por ELAIUY et al. (2009) e VALERO et al. (2009), dão evidências de que, além da água, a distribuição e o tamanho de partículas, e a superfície específica são características que podem influenciar no valor da constante dielétrica do material.

De acordo com os resultados da constante dielétrica aparente $(\mathrm{Ka})$ e da umidade, pelo método-padrão do bagaço de cana-de-açúcar $\left(\mathrm{m}^{3} \mathrm{~m}^{-3}\right)$, comprova-se existir uma associação linear entre ambas, apresentando um $\mathrm{R}^{2}$ próximo de 1 (Figura 9). Assim, com uma calibração, a técnica da TDR poderá ter um desempenho semelhante ao método-padrão para a obtenção dos valores da umidade em bagaço de cana-de-açúcar. Também se pôde obter uma equação linear de calibração. 


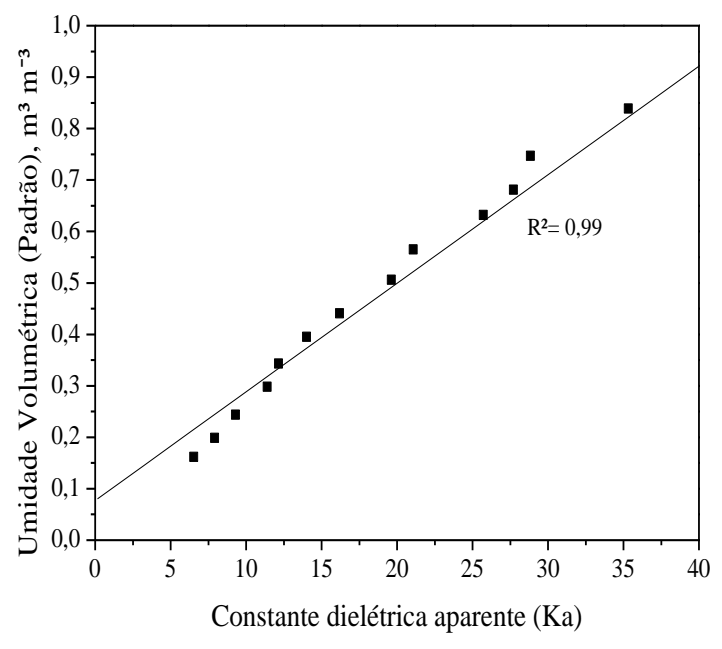

FIGURA 9. Calibração da sonda de TDR para bagaço de cana-de-açúcar, equação linear (Equação 2). TDR-probe calibration for cane sugar bagasse, linear equation (Equation 2).

Portanto, a Figura 10 apresenta o diagrama de dispersão e as curvas de regressão da umidade do solo em função da constante dielétrica aparente.

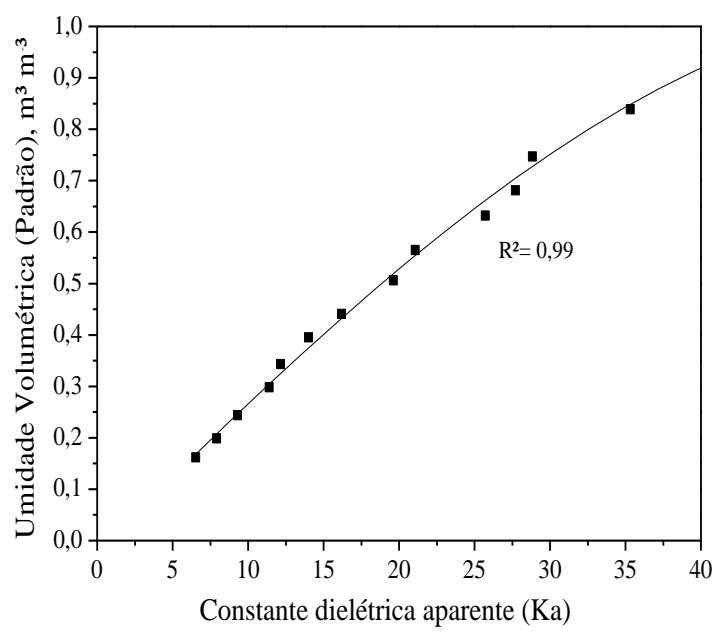

FIGURA 10. Calibração da sonda de TDR para bagaço de cana-de-açúcar, equação polinomial cúbica (Equação 3). TDR-probe calibration for cane sugar bagasse, cubic poly nomial equation (Equation 3).

Entre as equações de calibração, a mais indicada é a linear, pois envolve menor quantidade de fatores que podem ser sujeitos a erros. Com a inserção destas equações no software que acompanha a TDR, torna-se possível a estimativa da umidade volumétrica em bagaço de cana-de-açúcar pela técnica da TDR, a qual automatizará o monitoramento da umidade volumétrica do bagaço em tempo real, durante o processo biológico no reator.

$$
\begin{aligned}
& \theta=0,0253 \mathrm{Ka}-0,0025 \\
& \theta=4 * 10^{-6} \mathrm{Ka}^{3}-0,0005 \mathrm{Ka}^{2}+0,0385 \mathrm{Ka}-0,0704
\end{aligned}
$$

Com o objetivo de validar a equação encontrada, a Figura 11 apresenta o perfil de umidade do meio sólido durante o cultivo em estado sólido de Aspergillus niger, em bagaço de cana-de-açúcar impregnado com vinhaça. A partir dos dados, verifica-se uma queda na quantidade de água no sistema, mantendo-se, ainda assim, acima de um valor mínimo para crescimento de fungos filamentosos. 
Como o sistema foi alimentado com ar saturado, a redução na umidade pode ser atribuída pelo arraste de ar e pela condensação de água no topo da coluna. De fato, conforme indicado por LEKANDA \& PÉREZ-CORREA (2003), o acúmulo ou esgotamento de água no leito de sólidos depende da taxa de produção metabólica de água, das perdas por evaporação, da quantidade de água perdida no fundo da coluna e perdida na degradação do suporte sólido.

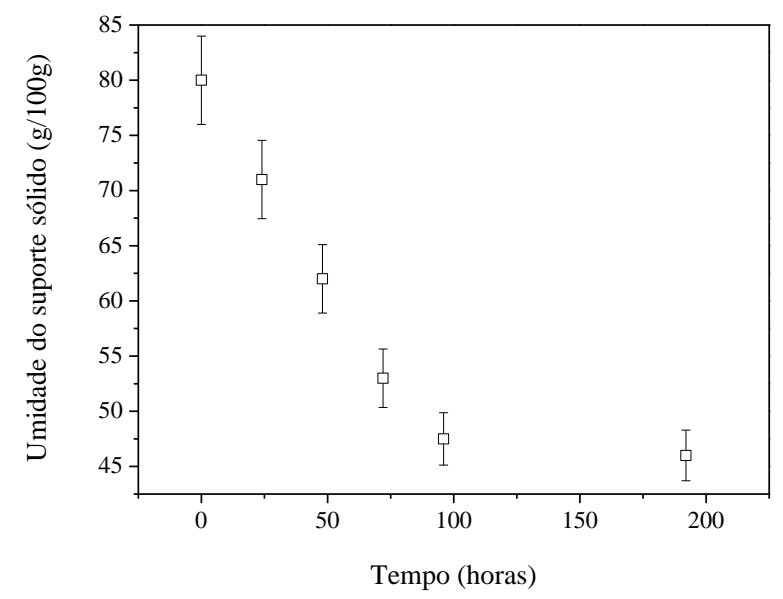

FIGURA 11. Perfil de umidade do meio sólido durante o cultivo de Aspergillus niger, em bagaço de cana-de-açúcar. Solid medium moisture profile for Aspergillus niger cultivation.

Como a estimativa de umidade pela TDR considera a medida média ao longo da coluna, este desconsidera possíveis gradientes de umidade que possam ocorrer ao longo do leito de partículas. Mesmo assim, o monitoramento em linha usando TDR é uma alternativa interessante para acompanhamento deste parâmetro fundamental, inclusive para o estudo do escalonamento destes biorreatores.

\section{CONCLUSÕES}

1. A sonda proposta apresenta o conjunto de critérios físicos que a qualifica como uma alternativa viável na obtenção da umidade em bagaço de cana-de-açúcar, através da técnica indireta da reflectometria no domínio do tempo.

2. A equação de calibração de Topp, que acompanha o equipamento (TDR 100), não é aplicável para estimativa de umidade volumétrica do bagaço da cana-de-açúcar; porém, se realizada uma calibração específica, a técnica da TDR mostrou-se viável para a estimativa da umidade em bagaço de cana-de-açúcar.

\section{AGRADECIMENTOS}

Ao CNPq, pela bolsa concedida ao primeiro autor, e à FAPESP (2011/07802-0), pelo financiamento do projeto.

\section{REFERÊNCIAS}

AOAC - ASSOCIATION OF OFFICIAL ANALYTICAL CHEMISTS. Official methods of analysis of the AOAC. $18^{\text {th }}$ ed. Gaithersburg, 2005.

BARROS, A.C.; FOLEGATTI, M.V.; SOUZA, C.F; SANTORO, B.L. Distribuição da solução no solo aplicado por gotejamento enterrado e superficial. Irriga, Botucatu, v.15, p.361-372, 2010.

BASTOS, R.G. Transferência de oxigênio no cultivo em estado sólido de Drechslera (Helminthosporium) monoce ras. 2006. 165 f. Tese (Doutorado em Engenharia de Alimentos) Universidade Estadual de Campinas, Faculdade de Engenharia de Alimentos, Campinas, 2006. 
BREUKELEN, F.R. van; HAEMERS, S.; WIJFFELS, R.H.; RINZEMA, A. Bioreactor and substrate selection for solid-state cultivation of the malaria mosquito control agent Metarhizium anisopliae. Process Biochemistry, New York, v.46, p.751-757, 2011.

BRUNHARA, J.P.B.; CIRILO, L.S.; SANTORO, B.L.; BERTOLETTE, H.P.; SOUZA, C.F. Manejo da fertirrigação por gotejamento na cultura do pimentão (Capsicum annum $L$ ) sob Latossolo vermelho escuro distrófico. In: CONGRESSO BRASILEIRO DE ENGENHARIA AGRÍCOLA, 40., 2011, Cuiabá. Anais... Cuiabá: Associação Brasileira de Engenharia Agrícola, 2011. 1 CD ROM.

ELAIUY, M.L.C.; SATO, L.M.; VARALLO, A.C.T.; SOUZA, C.F. Desenvolvimento e avaliação de sonda de TDR para o manejo racional da água em substratos utilizados na produção de mudas florestais. Revista Ambiente \& Água, Taubaté, v.4, p.117-131, 2009.

FASANELLA, C.P. Ação das enzimas ligninolíticas produzidas por Aspergillus niger e Penicillium sp. em bagaço de cana-de-açúcar tratado quimicamente. 2008. 81f. Dissertação (Mestrado em Microbiologia Agrícola) - Universidade de São Paulo, Escola Superior de Agricultura "Luiz de Queiroz", Universidade de São Paulo, Piracicaba, 2008.

HOFFMAN, G. Verbindliche methoden zur mntersuchung von TKS und gartnerischen erden. Mitteilungen der VDLUFA, v.6, p.129-153, 1970.

HORWITZ, W.; LATIMER, G. Official methods of analysis. $18^{\text {th }}$ ed. Washington: AOAC International, 2011. 2500p.

KUMAR, D.; JAIN, V.K. Solid state fermentation studies of citric acid. African Journal of Biotechnology, Nairobi, v.7, p.644-650, 2008.

LEKANDA, J.S.; PÉREZ-CORREA, J.R. Energy and water balances using kinetic modeling in a pilot-scale SSF bioreactor. Process Biochemistry, New York, v.39, p.1793-1802, 2003.

LOPES, L.N.; SOUZA, C.F.; S ANTORO, B.L. Utilização da TDR para monitoramento da solução de nitrato de potássio em Latossolo vermelho-amarelo. Engenharia Agrícola, Jaboticabal, v.30, p.932-947, 2010.

OLIVEIRA NETO, D.H.; CARVALHO, D.F.; SILVA, L.D.B.; GUERRA, J.G.M.; CEDDIA, M.B. Evapotranspiração e coeficientes de cultivo da beterraba orgânica sob cobertura morta de leguminosa e gramínea. Horticultura Brasileira, Brasília, v.29, p.330-334, 2011.

RÖBER, R.; SCHALLER, K. Pflazene rmahrung im gartenbau. Stuttgart: Ulmer, 1985. 350p.

SANTORO, B.L.; LOPES, L.N.; VARALLO, A.C.T.; SOUZA, C.F. Experiência do grupo de estudos em engenharia ambiental na confecção e utilização de sondas de TDR. In: SOUZA, C.F.; VARALLO, A.C.T.; LOPES, L.N.; SANTORO, B.L. Aplicações de técnicas eletromagnéticas para o monitoramento ambiental. Taubaté: UNITAU, 2008. p.185-227.

SANTOS, M.R.; ZONTA, J.H.; MARTINEZ, M.A. Influência do tipo de amostragem na constante dielétrica do solo e na calibração de sondas de TDR. Revista Brasileira de Ciência do Solo, Viçosa, v.34, p.299-307, 2010.

SILVA, B.M.; OLIVEIRA, G.C.; SERAFIM, M.E.; SILVA JÚNIOR, J.J.; COLOMBO, A.; LIMA, J.M. Acurácia e calibração de sonda de capacitância em Latossolo Vermelho cultivado com cafeeiro. Pesquisa Agropecuária B rasileira, Brasília, v.47, p. 277-286, 2012.

SINGHANIA, R.R.; PATEL, A.K.; SOCCOL, C.R.; PANDEY, A. Recent advances in solid-state fermentation. Biochemical Engineering Journal, New York, v.44, p.13-18, 2009.

SONCELA, R.; SAMPAIO, S.C.; VILAS BOAS, M.A.; TAVARES, M.H.F.; SOUZA, C.F.; Soncela, A. S. Electrical conductivity of soil irrigated with swine wastewater estimated by timedomain reflectometry. Revista Brasileira de Ciência do Solo, Viçosa, v.35, p.1293-1300, 2011. 
SOUZA, C.F.; FOLEGATTI, M.V. Spatial and temporal characterization of water and solute distribution patterns. Scientia Agricola, Piracicaba, v.67, p.9-15, 2010.

SOUZA, C.F.; FOLEGATTI, M.V.; OR, D. Distribution and storage characterization of soil solution for drip irrigation. Irrigation Science, Heidelberg, v.27, p.277-288, 2009.

VALERO, R.M.M.; MATSURA, E.E.; SOUZA, A.L. Caracterização física de dois substratos orgânicos para plantas e a estimativa da umidade por meio da reflectometria no domínio do tempo. Ciência Rural, Santa Maria, v.39, p.571-574, 2009. 